\title{
Síntesis y caracterización de películas delgadas de SnS-SnSe de estructura cristalina cubica crecidas por baño químico
}

\author{
E. Barrios-Salgado ${ }^{a^{*}}$, J. P. Pérez-Orozco ${ }^{b}$ y Y. Rodríguez-Lazcano ${ }^{a}$ \\ ${ }^{a}$ Universidad Autónoma de Nayarit, Ciudad de la Cultura "Amado Nervo" S/N, C.P. 63155, Tepic, Nayarit, México. \\ ${ }^{b}$ Instituto Tecnológico de Zacatepec, Departamento de Ingeniería Química y Bioquímica, Calzada Tecnológico No. 27, Col. Centro \\ Zacatepec, Morelos 62780, México. \\ *E-mail: ebs@ier.unam.mx
}

Recibido 12 noviembre 2016, Aceptado 20 diciembre 2016

\section{Resumen}

Recientemente han reportado que es posible crecer películas delgadas de sulfuro de estaño (SnS) y selenuro de estaño ( $\mathrm{SnSe}$ ) con estructura ortorrómbica sobre películas delgadas de $\mathrm{SnS}$ de estructura cúbica. En el presente trabajo se muestra cómo se puede llevar a cabo la transformación de la estructura cristalina de las películas delgadas de $\mathrm{SnSe}$ y $\mathrm{SnS}$ de ortorrómbica a cubica. Se presenta la metodología de depósito para la formación de las películas delgadas, así como las características estructurales, ópticas y eléctricas de las películas preparadas sin tratamiento térmico (TT) y con tratamiento térmico a $240{ }^{\circ} \mathrm{C}$ en atmósfera de nitrógeno a 10 Torr durante 30 min. Para mostrar el cambio de estructura cristalina, así como el efecto del TT en las películas formadas, se presentan los espectros de difracción de rayos X (DRX), la transmitancia $(\mathrm{T})$, reflectancia $(\mathrm{R})$ y fotorrespuesta. Se determinó una brecha de energía $\left(\mathrm{E}_{\mathrm{g}}\right)$ de $1.2 \mathrm{eV}$ y conductividad eléctrica $(\sigma)$ de $1.88 \times 10^{-4} \Omega^{-1} \cdot \mathrm{cm}^{-1}$ para la película sin TT, para la película horneada en $\mathrm{N}_{2}$ se determinó una $\mathrm{E}_{\mathrm{g}}$ de $1.4 \mathrm{eV}$ y $\sigma$ de $5.8 \times 10^{-3} \Omega^{-1} \cdot \mathrm{cm}^{-1}$. Mediante la punta caliente se estableció que las películas son de conductividad tipo-p. Estas características ópticas y eléctricas junto con la abundancia de estaño ( $\mathrm{Sn}$ ) en la corteza terrestre hacen de este material una excelente alternativa para su aplicación como absorbedor en celdas solares de bajo costo.

Palabras clave: $\mathrm{SnSe}, \mathrm{SnS}$, películas delgadas, absorbedor.

\section{Introducción}

En los últimos años la investigación sobre los semiconductores calcogenuros de estaño, tales como, $\mathrm{SnS}$ y $\mathrm{SnSe}$ ha crecido considerable, debido a que se ha mostrado que éstos presentan características optoelectrónicas que permiten su aplicación tanto en la tecnología fotovoltaica como en la termoeléctrica. Se han reportado técnicas de depósito tanto físicas como químicas para la formación de estos materiales en forma de película delgada y nanocristales. Por baño químico existen diversas metodologías de depósito y dependiendo de la composición química del baño, así como de la temperatura de depósito se han obtenido películas de $\mathrm{SnS}$ y SnSe con estructura cristalina cubica u ortorrómbica [14]. En el 2015 y 2016 reportaron una nueva estructura cristalina para estos materiales en forma de película delgada, así como en nanocristales, la nueva estructura es una cúbica larga cuya celda unitaria consta de 32 unidades de $\mathrm{SnS}$ o 64 átomos, esto es que el borde del cubo es el doble de una cubica sencilla [5-8]. Películas delgadas de $\mathrm{SnS}$ y $\mathrm{SnSe}$ elaboradas por baño químico se han identificado como cúbicas largas con parámetros de red de $11.9632 \AA$ y $11.5873 \AA[7,8]$. Estas películas presentan brechas de energía entre 1.2 y $1.4 \mathrm{eV}$ y conductividad eléctrica del orden de $10^{-2}$ y $10^{-4} \Omega^{-1} \cdot \mathrm{cm}^{-1}$, las variaciones de Eg y conductividad eléctrica están en función del espesor, así como de los tratamientos postdepósitos de las películas [7,8]. Los trabajos ya reportados sobre las películas con la estructura cúbica larga, para la obtención del SnS-CUB se han usado como fuente de $\mathrm{Sn}$ el cloruro de estaño $\left(\mathrm{SnCl}_{2} \cdot 2 \mathrm{H}_{2} \mathrm{O}\right)$ y como fuente de azufre una solución de $\mathrm{Sn}$ (II) o tioacetamida $\left(\mathrm{CH}_{3} \mathrm{CSNH}_{2}\right)$, cuya metodología se encuentra detallada en la referencia [9]. Dado a que la nueva estructura cristalina muestra características prometedoras para su aplicación en celdas solares, en el presente trabajo hemos investigado una nueva metodología para la formación de películas delgadas de $\mathrm{SnS}-\mathrm{SnSe}$ con estructura cubica larga, formadas por la técnica de depósito por baño químico (DBQ). Son varios los factores que se deben cuidar en la técnica de DBQ para lograr formar películas delgadas de buena calidad, la concentración de las soluciones químicas, la temperatura de depósito y el $\mathrm{pH}$ de la solución, así como la superficie sobre la cual se deposita la película. Se ha mostrado que el tratamiento de los sustratos en una solución de sulfuro de sodio mejora significativamente la calidad de la película [4]. Es importante controlar el $\mathrm{pH}$ de la solución del baño, esto debido a que la técnica de DBQ consiste en la inmersión de sustratos a una solución con un determinado $\mathrm{pH}$ que en ocasiones puede disolver la película previa, cuando se 
es necesario depositar, por ejemplo: películas de forma estratificada para formar compuestos ternarios $o$ cuaternarios, o bien, para cuando se fabrican celdas de tipo superestrato y el absorbedor es depositado por la técnica de DBQ. En varios casos las celdas fabricadas por DBQ se van a corto circuito debido a que la solución del absorbedor disuelve la película previa y no se logra una buena unión n-p, propiciando así que la respuesta del efecto fotovoltaico sea casi nula.

Aquí se realizan depósitos secuenciales para obtener películas delgadas de $\mathrm{SnS}-\mathrm{SnSe}$ de estructura cúbica larga, a continuación, se describe la metodología, así como algunos resultados preliminares obtenidos.

\section{Parte experimental}

Para el depósito de las películas delgadas se utilizaron como sustratos de vidrio portaobjetos (marca Corning), con dimensiones de $25 \mathrm{~mm}$ x $75 \mathrm{~mm}$ x $1 \mathrm{~mm}$. Los sustratos fueron sometidos a un proceso rígido de limpieza, utilizando gasa humedecida con una solución de detergente y después enjuagando con agua corriente y agua destilada. Finalmente se secaron con aire caliente y se dejaron enfriar a temperatura ambiente. Posteriormente los sustratos fueron sumergidos en una solución de sulfuro de sodio $3 \mathrm{M}$ contenido en un vaso de precipitados de $100 \mathrm{ml}$ durante $1 \mathrm{~h}$ a $80^{\circ} \mathrm{C}$, pasado este tiempo los sustratos fueron retirados de la solución, se enjuagaron con agua destilada y se secaron con aire caliente. Este pre-tratamiento de los sustratos permite crecer películas delgadas $\mathrm{SnS}$ y $\mathrm{SnSe}$ de mejor calidad y con características reproducibles. Para el control de la temperatura de los baños químicos se usó un baño de circulación de la marca Polyscience.

Los sustratos pre-tratados se colocaron verticalmente en un vaso de precipitados de $100 \mathrm{ml}$ el cual contiene la solución del baño de depósito correspondiente para cada película. Al final de la duración deseada de los depósitos, los sustratos se sacan del baño, se enjuagan con agua destilada y se secan. El método de depósito por inmersión en la solución, comúnmente empleado en el baño químico, produce películas sobre ambas caras del sustrato. El depósito en la cara cercana de la pared del vaso de precipitado es, generalmente, más uniforme y presenta reflexiones características de la película delgada. Para realizar la medición de espectros ópticos se removió la película que crece de lado opuesto a la pared del vaso con un cotonete humedecido con $\mathrm{HCl}$ diluido.

Para el depósito de las películas delgadas de SnS-CUB se siguió una metodología previamente reportada [9]. En un vaso de precipitados de $100 \mathrm{ml}$ se vertieron, $10 \mathrm{ml}$ de una solución de Sn (II) $0.1 \mathrm{M}, 30 \mathrm{ml}$ de trietanolamina (TEA) $3.7 \mathrm{M}, 16 \mathrm{ml}$ de hidróxido de amonio $\left(\mathrm{NH}_{4} \mathrm{OH}\right) \sim 15 \mathrm{M}$, $10 \mathrm{ml}$ de tioacetamida $\left(\mathrm{C}_{2} \mathrm{H}_{5} \mathrm{NS}\right) 0.1 \mathrm{M}$, y para completar un volumen de $100 \mathrm{ml}$ se vertieron $34 \mathrm{ml}$ de agua destilada. La duración del depósito fue de $6 \mathrm{~h}$ a $25^{\circ} \mathrm{C}$. El espesor de la película a estas condiciones de depósito es de $\sim 120 \mathrm{~nm}$
Sobre las películas de SnS-CUB de $120 \mathrm{~nm}$ de espesor, se realizó el depósito de las películas delgadas de SnSORT, la composición química del baño ha sido reportada previamente [1]. En un vaso de precipitados de $100 \mathrm{ml} \mathrm{se}$ vertieron unas gotas de ácido clorhídrico para disolver el cloruro de estaño $\left(\mathrm{SnCl}_{2}\right)$, la concentración molar del $\mathrm{SnCl}_{2}$ en el volumen total es de $0.03 \mathrm{M}$. A continuación, se adicionó ácido tartárico $\left(\mathrm{C}_{4} \mathrm{H}_{6} \mathrm{O}_{6}\right)$ para tener una concentración molar de $0.44 \mathrm{M}$ de $\mathrm{SnCl}_{2}$ de en el volumen total. Se añadió agua destilada, posteriormente $\mathrm{NH}_{4} \mathrm{OH}$ para obtener el $\mathrm{pH}$ de la solución igual a 7. El último componente añadido fue el tiosulfato de sodio $\left(\mathrm{Na}_{2} \mathrm{~S}_{2} \mathrm{O}_{3}\right)$ con una concentración molar de $0.03 \mathrm{M}$ en volumen total de $100 \mathrm{ml}$. El depósito se llevó a cabo a durante $20 \mathrm{~h}$ a $17^{\circ} \mathrm{C}$. El espesor de la película delgada de $\mathrm{SnS}-\mathrm{CUB} / \mathrm{SnS}-\mathrm{ORT}$ es de $\sim 250 \mathrm{~nm}$.

Posteriormente sobre la película delgada de $\mathrm{SnS}-$ CUB/SnS-ORT de $250 \mathrm{~nm}$ de espesor, se depositó una película de SnSe durante $3 \mathrm{~h}$ a $30^{\circ} \mathrm{C}$, para producir una película de $\mathrm{SnS}-\mathrm{CUB} / \mathrm{SnS}-\mathrm{ORT} / \mathrm{SnSe}-$ de $\sim 300 \mathrm{~nm}$ de espesor. La composición química del baño de $\mathrm{SnSe}$ ha sido previamente reportada [4]. En un vaso de precipitados de $100 \mathrm{ml}$ se vertieron, $0.7 \mathrm{~g}$ de $\mathrm{SnCl}_{2}$ y se disolvieron con $5 \mathrm{ml}$ de acetona, posteriormente se agregaron $35 \mathrm{ml}$ de TEA $3.5 \mathrm{M}, 18 \mathrm{ml}$ de hidróxido de $(\mathrm{NaOH}) \quad 2.0 \quad \mathrm{M}, \quad 0.25 \quad \mathrm{ml}$ una solución de polivinilpirrolidona (PVP) al $0.5 \%, 4.0 \mathrm{ml}$ de selenosulfato de sodio $\left(\mathrm{Na}_{2} \mathrm{SeSO}_{3}\right) 0.2 \mathrm{M}$, y $16 \mathrm{ml} \mathrm{de}$ agua destilada.

Finalmente, con el objetivo de mejorar y observar cualquier cambio en la composición química o estructura cristalina de las películas delgadas obtenidas y por lo tanto evaluar la estabilidad térmica de éstas, se realizó un tratamiento térmico a $240{ }^{\circ} \mathrm{C}$ durante $30 \mathrm{~min}$ en nitrógeno, $\mathrm{N}_{2}$ (a 10 Torr) en un horno de vacío (High Vacuum Equipment Riverton).

Para la caracterización estructural las películas delgadas, se utilizó un difractómetro ULTIMA IV con radiación $\mathrm{K}_{\alpha}$ del cobre $((\lambda \mathrm{Cu}=1.5405 \AA)$ en ángulo de incidencia rasante $(\delta)$ de $2^{\circ}$ con el plano de la muestra. Los datos fueron analizados para evaluar los tamaños del cristal en las películas delgadas y el parámetro de red. Los espectros de transmitancia $(\mathrm{T})$ y reflectancia $(\mathrm{R})$ fueron obtenidos en un espectrofotómetro Shimadzu UV3101PC UV-VIS-NIR, en el intervalo entre los 250 y $2500 \mathrm{~nm}$. Para la T se utilizó aire como referencia y para la R un espejo aluminizado. Para medir la fotorrespuesta, se pintaron sobre la superficie de las películas electrodos de carbón, usando pintura de grafito coloidal (SPIChem). El tamaño del electrodo fue de $5 \mathrm{~mm}$ de largo por $5 \mathrm{~mm}$ de separación aproximadamente. La corriente en oscuridad y bajo iluminación se midió en un sistema computarizado usando un electrómetro Keithley 619 y una fuente de voltaje programable Keithley 230. Todas las mediciones se realizaron manteniendo la muestra durante 20 segundos en oscuridad, seguidamente 20 segundos bajo iluminación y finalmente 20 segundos más en oscuridad. Las mediciones de fotorrespuesta fueron realizadas a temperatura ambiente y presión atmosférica 
utilizando una lámpara de halógeno-tungsteno, que proporciona una intensidad de luz de $800 \mathrm{~W} / \mathrm{m}^{2}$ sobre la superficie de la muestra. La conductividad eléctrica se obtiene a partir de los valores medidos de corriente y voltaje. Para determinar el tipo de conductividad de las películas se usó la técnica de la punta caliente. La punta caliente adopta potencial positivo sobre el material tipo-n y negativo sobre el material tipo-p, cuando la conductividad eléctrica es típicamente $>10-4(\Omega \cdot \mathrm{cm})-1$. Para materiales menos conductivos la determinación tiene incertidumbre.

\section{Resultados y discusión}

En la Figura 1 se muestran los DRX con incidencia de haz rasante de $2^{\circ}$ para las películas delgadas desarrolladas. En la parte inferior y superior de esta figura se muestran los patrones estándar del $\mathrm{SnS}$ ortorrómbico (PDF 39-0354) y los correspondientes a las fases cubicas larga del SnS-CUB y SnSe-CUB, respectivamente. En el inciso a) se muestra el patón de DRX correspondiente a la película estratificada de SnSCUB/SnS-ORT, en la cual se observan picos de difracción de los planos (101), (111), (040), (041) y (002) correspondientes a la fase ortorrómbica del $\mathrm{SnS}$, comparables con el patrón estándar PDF 39-0354 asignado para el $\mathrm{SnS}$ de estructura cristalina ortorrómbica con parámetros de red $\mathrm{a}=4.3293$, $\mathrm{b}=11.1923$ y $\mathrm{c}=3.9838 \AA$, y picos asociados a la fase cubica larga (222), (400) y (410). En los incisos b) y c) se muestran los DRX de las películas estratificadas de $\mathrm{SnS}-$ CUB/SnS-ORT/SnSe- sin TT y con TT a $240{ }^{\circ} \mathrm{C}$, respectivamente. Aquí podemos observar principalmente los picos de difracción de los planos (222), (400), (410) y (411) correspondientes a las fases de SnS-CUB y SnSeCUB larga. Cuyos parámetros de red son de $11.5873 \AA$ y $11.9632 \AA$, para el SnS y SnSe, respectivamente. En los incisos b) y c) es evidente el cambio en las intensidades de los planos (410) y (400) en las posiciones 31.8 y $30.82^{\circ}$, respectivamente. Este cambio en las intensidades puede asociarse al cambio de fase de ortorrómbico a cúbico. Si bien en el inciso d) no se observa claramente el desplazamiento de los planos hacia la izquierda, en donde se espera mayor cantidad de $\mathrm{SnSe}$, la relación de las intensidades de los planos (222), (400) y (410) son comparables con las del patrón asignado para el SnSeCUB. Lo cual nos sugiere que probablemente incrementado la duración del depósito de SnSe o bien la reducción del tiempo de depósito del SnS-ORT podría definirse la fase del SnSe-CUB totalmente. Por otro lado, el tratamiento térmico a $240{ }^{\circ} \mathrm{C}$ realizado a la película nos indica que la muestra es estable, tal como se observa en los incisos b) y c), no se tiene variación en las intensidades de los picos y no se observa perdida del material. El tamaño promedio del cristal de estas películas obtenidas es de $\sim 20 \mathrm{~nm}$.

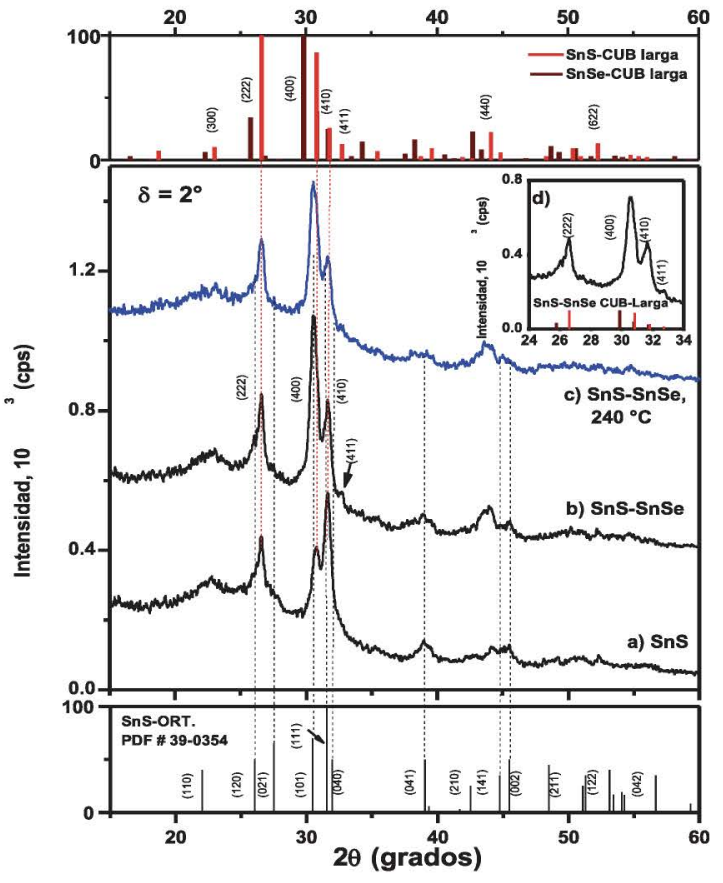

Figura 1. DRX $\left(\delta=2^{\circ}\right)$ de las películas delgadas crecidas de forma estratificada por DBQ: a) SnS-CUB/SnS-ORT, b) SnSCUB/SnS-ORT-SnSe-CUB sin TT y c) SnS-CUB/SnS-ORTSnSe-CUB con TT a $240^{\circ} \mathrm{C}$ durante 30 min en $\mathrm{N}_{2}$ a 10 Torr. Se incluyen los patrones estándar del SnS-ORT, SnS-CUB y SnSe-CUB (cubica larga).

En la Figura 2 se presentan el coeficiente de absorción óptico $\left(\alpha, \mathrm{cm}^{-1}\right)$ de las películas delgadas de elaboradas como una función de la energía del fotón $(\mathrm{hv}, \mathrm{eV})$ el cual es evaluado a partir de los valores de $\mathrm{T}$ y $\mathrm{R}$, utilizando la ecuación (1) de múltiples reflexiones en películas delgadas [10]:

$$
\alpha=\frac{(1-\mathrm{R})^{4}+\sqrt{(1-R)^{4}+(2 R T)^{2}}}{2 T}
$$

donde $\mathrm{T}$ es la transmitancia, $\mathrm{R}$ es la reflectancia, $\mathrm{y} \mathrm{d}$ es el espesor de la película.

A partir de la curva de $\alpha$ se estima una $\mathrm{E}_{\mathrm{g}}$ de $1.7 \mathrm{eV}$ para la película delgada de SnS-CUB/SnS-ORT de $250 \mathrm{~nm}$ de espesor (ver fig. 2 a)), este valor es comparable con 1.73 reportado previamente para películas delgadas de $\mathrm{SnS}$ de estructura cubica simple obtenidas por DBQ [9], y al reportado de 1.66 a $1.72 \mathrm{eV}$ para las películas delgadas de $\mathrm{SnS}$ de estructura cubica larga obtenida por la misma técnica [8]. De los incisos b) y c) de la figura 2, se estimó una $\mathrm{E}_{\mathrm{g}}$ de $1.63 \mathrm{eV}$, para las películas de $\mathrm{SnS}-\mathrm{CUB} / \mathrm{SnS}$ $\mathrm{ORT} / \mathrm{SnSe}-$ con y sin TT. La $\mathrm{E}_{\mathrm{g}}$ es directa con transición óptica prohibida ya que la variación de $(\alpha h v)^{2 / 3}$ contra hv fue la curva que dio el mejor ajuste lineal para determinar $\mathrm{E}_{\mathrm{g}}$. Estos resultados son coherentes, además de que están acorde a lo reportado previamente, es claro que las películas con o sin TT son estables y no se observan cambios ópticos, lo cual confirma lo observado en los 
DRX. La reducción de la $\mathrm{E}_{\mathrm{g}}$ que se observa es esperada, esto debido a la incorporación de la película delgada de SnSe en SnS-CUB/SnS-ORT, conociendo que el SnSe de estructura cubica larga se ha reportado una $\mathrm{E}_{\mathrm{g}}$ de $1.4 \mathrm{eV}$, se esperaba un valor de $\mathrm{E}_{\mathrm{g}}$ dentro de $1.4 \mathrm{a} 1.7 \mathrm{eV}$. Estos resultados nos permiten aseverar la formación de la fase cubica larga, esto porque las fases ortorrómbicas del $\mathrm{SnS}$ y $\mathrm{SnSe}$ en forma de película delgada reportado es de $\sim 1$ $\mathrm{eV}[4,8]$.

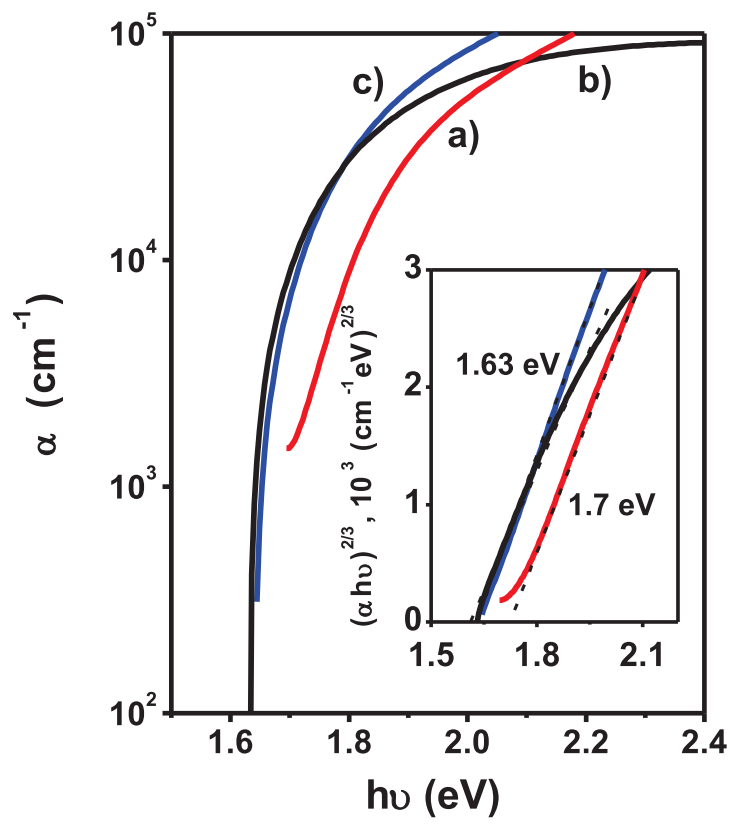

Figura 2. Coeficiente de absorción óptico de las películas delgadas de: a) SnS-CUB/SnS-ORT de $250 \mathrm{~nm}$ de espesor, b) $\mathrm{SnS}-\mathrm{CUB} / \mathrm{SnS}-\mathrm{ORT} / \mathrm{SnSe}-$ de $300 \mathrm{~nm}$ de espesor sin TT y c) $\mathrm{SnS}-\mathrm{CUB} / \mathrm{SnS}$-ORT/SnSe- de $300 \mathrm{~nm}$ de espesor con TT en $\mathrm{N}_{2}$ a $240{ }^{\circ} \mathrm{C}$; e insertadas las curvas de $\mathrm{E}_{\mathrm{g}} \mathrm{vs}(\alpha \mathrm{hv})^{2 / 3}$

En la Figura 3 a) se muestra la fotoconductividad de las películas delgadas de SnS-CUB/SnS-ORT de $250 \mathrm{~nm}$ de espesor, la conductividad eléctrica en oscuridad $\left(\sigma_{\text {osc. }}\right)$ y bajo iluminación $\left(\sigma_{\text {ilum }}\right)$ es de $1.23 \times 10^{-4} \Omega^{-1} \cdot \mathrm{cm}^{-1}$ y $2.54 \times 10^{-4} \Omega^{-1} \cdot \mathrm{cm}^{-1}$, respectivamente. En los incisos b) y c) se muestra la fotoconductividad de las películas delgadas de SnS-CUB/SnS-ORT/SnSe- de $300 \mathrm{~nm}$ de espesor sin tratamiento térmico y con tratamiento térmico, respectivamente. La conductividad eléctrica de estas películas difiere por una orden de magnitud, de $10^{-4}$ a $10^{-}$ ${ }^{3} \Omega^{-1} \cdot \mathrm{cm}^{-1}$, siendo más conductiva la película horneada en atmosfera de $\mathrm{N}_{2}$ a $240{ }^{\circ} \mathrm{C}$ durante $30 \mathrm{~min}$. El aumento paulatino que se observa en la fotoconductividad es debido al aumento de la movilidad de portadores de carga. Estos resultados son buenos, generalmente para un material absorbedor para su uso en celdas solares se busca que la conductividad eléctrica sea de estos órdenes de magnitud.

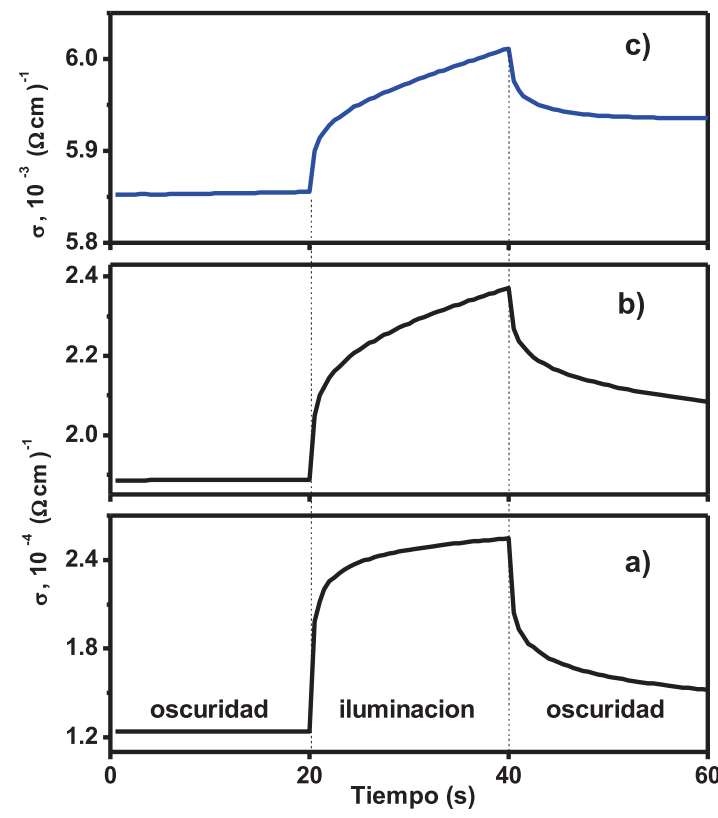

Figura 3. Fotorrespuesta de las películas delgadas de: a) SnSCUB/SnS-ORT de $250 \mathrm{~nm}$ de espesor, b) SnS-CUB/SnSORT/SnSe- de $300 \mathrm{~nm}$ de espesor sin TT y c) SnS-CUB/SnSORT $/ \mathrm{SnSe}$ - de $300 \mathrm{~nm}$ de espesor con TT en $\mathrm{N}_{2}$ a $240{ }^{\circ} \mathrm{C}$.

\section{Conclusiones}

En el presente trabajo se propone una nueva metodología para convertir películas delgadas de $\mathrm{SnS}-\mathrm{SnSe}$ de estructura ortorrómbica a estructura cúbica larga con parámetros de red $11.9632 \AA$ y $11.5873 \AA$, es decir, que la celda unitaria consta en 32 unidades o 64 átomos. El método consiste en realizar depósitos químicos secuenciales de SnS-CUB, SnS-ORT y SnSe. Cuando se realiza el depósito químico del $\mathrm{SnSe}$ sobre el $\mathrm{SnS}$ CUB/SnS-ORT se observa claramente el cambio de orientación en los planos principales de la fase ortorrómbica a la cúbica larga. Estas películas presentan una $\mathrm{E}_{\mathrm{g}}$ de $1.63 \mathrm{eV}$ y $1.7 \mathrm{eV}$, correspondiendo el valor menor a la película con $\mathrm{SnSe}$, las conductividades eléctricas de estas películas son del orden de $10^{-4}$ a $10^{-3}$ $\Omega^{-1} \cdot \mathrm{cm}^{-1}$. Mediante la punta caliente se determinó que las películas son de conductividad tipo-p. Se mostró que las películas son estables a temperaturas de $240{ }^{\circ} \mathrm{C}$, ya que no se observan cambios estructurales y ópticos. Estas características hacen de este material potencial candidato para su uso en celdas solares de bajo costo. Estos resultados son preliminares, actualmente se investigan métodos de preparación que permitan mejorar la cristalinidad de la película.

\section{Agradecimientos}

Al CONACyT-LIFYCS, al IER-UNAM por todas las facilidades brindadas en el uso de sus instalaciones para la realización de la caracterización eléctrica y estructural de las películas delgadas desarrolladas. Al programa de Repatriación y Retención del CONACyT, por la repatriación de Y.R.L y retención de E.B.S. 


\section{Referencias}

1. Safonova M., Nair P. K., Mellikov E., Garcia A. R., Kerm K., Revathi N., Romann T., Mikli V. Volobujeva O. J Mater Sci: Mater Electron 2014, 25, 3160-3165.

2. Jayasree Y., Chalapathi U., Bhaskar P. U., Raja V. S. Applied Surface Science 2012, 258, 2732- 2740 .

3. Garcia-Angelmo A. R., Nair M. T. S., Nair P. K. Solid State Sci. 2014, 30, 26-35.

4. Barrios-Salgado E., Nair M.T.S., Nair P.K. ECS J. Solid State Sci. Technol. 2014, 3, Q169-Q175.

5. Rabkin A., Samuha S., Abutbul R. E., Ezersky V., Meshi L., Golan Y. Nano Lett. 2015, 3, 2174-2179.

6. Abutbul R. E., Segev E., Zeiri L., Ezersky V., Makov G., Golan Y. RSC Adv. 2016, 6 58485855.

7. Nair P. K., Barrios-Salgado E., Nair M. T. S. Phys. Status Solidi A 2016, 1-8. DOI 10.1002/pssa. 201533040

8. Nair P. K., Garcia-Angelmo A. R. Nair., M. T. S. Phys. Status Solidi A 2016, 213, 170-177.

9. Avellaneda D., Nair M. T. S. Nair P. K. J. Electrochem. Soc. 2008, 155, D517-D525.

10. Schröder D.K., 2006, Semiconductor Material and Device Characterization, third ed., New York: John Wiley \& Sons. 